\title{
Hyperbolic Shear Polaritons in Low-Symmetry Crystals
}

\section{Nikolai Passler}

Fritz-Haber-Institut der Max-Planck-Gesellschaft

\section{Xiang Ni}

The City College Of New York

\section{Guangwei Hu}

National University of Singapore https://orcid.org/0000-0002-3023-9632

\section{Joseph Matson}

Vanderbilt University

\section{Martin Wolf}

Fritz-Haber-Institut der May-Planck-Gesellschaft

\section{Mathias Schubert}

University of Nebraska

\section{Andrea Alu}

CUNY Advanced Science Research Center https://orcid.org/0000-0002-4297-5274

\section{Joshua Caldwell}

Vanderbilt University, Mechanical Engineering Department https://orcid.org/0000-0003-0374-2168

Thomas Folland

University of lowa https://orcid.org/0000-0002-4665-235X

\section{Alexander Paarmann ( $\square$ alexander.paarmann@fhi-berlin.mpg.de )}

Fritz Haber Institute of the Max Planck Society https://orcid.org/0000-0002-8271-2284

\section{Physical Sciences - Article}

Keywords: polaritons, photonic devices, crystals

Posted Date: June 21st, 2021

DOl: https://doi.org/10.21203/rs.3.rs-558805/v1

License: (a) (i) This work is licensed under a Creative Commons Attribution 4.0 International License. Read Full License 
Version of Record: A version of this preprint was published at Nature on February 23rd, 2022. See the published version at https://doi.org/10.1038/s41586-021-04328-y. 


\title{
Hyperbolic Shear Polaritons in Low-Symmetry Crystals
}

\author{
Nikolai C. Passler, Xiang Ni, Guangwei Hu, Joseph R. Matson, Martin Wolf, \\ Mathias Schubert, Andrea Alù, Joshua D. Caldwell, Thomas G. Folland, \\ Alexander Paarmann
}

The lattice symmetry of a crystal is one of the most important factors in determining its physical properties. Particularly, low-symmetry crystals offer powerful opportunities to control light propagation, polarization and phase ${ }^{1-4}$. Materials featuring extreme optical anisotropy can support a hyperbolic response, enabling coupled light-matter interactions, also known as polaritons, with highly directional propagation and compression of light to deeply sub-wavelength scales ${ }^{5,6}$. Here we show that monoclinic crystals can support hyperbolic shear polaritons, a new polariton class arising in the mid- to far-infrared due to shear dissipation in the dielectric response. This feature emerges in materials where the dielectric tensor cannot be diagonalized, that is, in low-symmetry monoclinic and triclinic crystals where multiple oscillators with non-orthogonal relative orientations contribute to the optical response ${ }^{7,8}$. Hyperbolic shear polaritons complement previous observations of hyperbolic phonon polaritons in orthorhombic ${ }^{1,3,4}$ and hexagonal ${ }^{9-11}$ crystal systems, unveiling new features, such as the continuous evolution of their propagation direction with frequency, tilted wavefronts and asymmetric responses. The interplay between diagonal loss and off-diagonal shear dissipation in the dielectric response of these materials has implications for new forms of non-Hermitian and topological photonic states. We anticipate that our results will motivate new directions for polariton physics in low-symmetry materials, which include geological minerals ${ }^{12}$, many common oxides ${ }^{13,14}$ and organic crystals ${ }^{15}$, significantly expanding the material base and extending design opportunities for compact photonic devices.

Crystal symmetry plays a critical role in dictating the optical, electronic, mechanical and thermal properties of a material. Reduced symmetry is at the heart of numerous emergent phenomena, including structural phase transitions ${ }^{13}$, charge density waves ${ }^{16,17}$ and topological physics ${ }^{18}$. The interaction of light with low-symmetry materials is particularly important, as it allows fine control over the phase, propagation direction and polarization ${ }^{1-4}$. This control can be especially pronounced for sub-diffractional surface waves, for instance surface phonon polaritons (SPhPs) ${ }^{19,20}$ and surface plasmon polaritons (SPPs), supported at the surface of polar crystals and conductors, respectively. Both SPhPs and SPPs are quasi-particles comprised of photons and coherently oscillating charges, i.e., polar lattice vibrations or free-carrier plasmas, respectively, and they are strongly influenced by crystal symmetry. As a relevant example, low-symmetry polaritonic materials can support hyperbolic light propagation ${ }^{21}$, constituting an exotic class of light waves that are highly directional with very large momenta. Hyperbolic polaritons arise in materials where the real part of the permittivity along at least one crystal direction is negative, while positive along at least one other, stemming from the extreme anisotropy of their response typically associated with specifically polarized lattice resonances. In turn, hyperbolic polaritons enable deeply subwavelength light confinement over broad bandwidths ${ }^{9-11}$. In polar crystals with symmetries that support a single optical axis (uniaxial), such as hexagonal boron nitride (hBN), hyperbolic polaritons (hPs) of type I or II can arise ${ }^{5,9-11}$, for which the hyperbolic isofrequency surfaces do or do not intersect the optical axis, respectively. Materials or metamaterials exhibiting lower symmetry, where all three major polarizability axes are different (biaxial) but orthogonal, such as alpha-phase molybdenum trioxide $\left(\alpha-\mathrm{MoO}_{3}\right)^{3,22}$, Liintercalated $\mathrm{V}_{2} \mathrm{O}_{5}{ }^{4}$, or nanostructured metasurfaces ${ }^{23}$, exhibit several distinct spectral regimes of hyperbolic modes propagating along different crystal axes. Notably, in-plane hyperbolicity within $\alpha-$ $\mathrm{MoO}_{3}$ films has been shown to be low-loss ${ }^{3,22}$, with reconfigurable features ${ }^{24}$ and capable of 
supporting topological transitions ${ }^{2}$. Even more exotic polaritonic responses may be expected in crystals with further reduced symmetry, such as monoclinic and triclinic lattices.

Monoclinic crystals make up the largest crystal system, with approximately one third of Earth's minerals belonging to one of its three classes ${ }^{25}$. These low-symmetry Bravais lattices exhibit nonorthogonal principal crystal axes (Fig. 1a), in contrast to orthorhombic (e.g. biaxial $\alpha-\mathrm{MoO}_{3}{ }^{1}$ ), tetragonal, hexagonal, trigonal (e.g. uniaxial $\alpha$-Quartz, Fig. 1b), or cubic crystal systems. As a consequence, their dielectric permittivity tensor has major polarizability directions that strongly depend on the frequency, with off-diagonal terms that cannot be completely removed through coordinate rotation ${ }^{7,8}$. These features arise due to the non-trivial relative orientation (neither parallel nor orthogonal) of multiple optical transitions that, at a given frequency, contribute to a net polarization that cannot be aligned with the crystal axes. In turn, this property results in exotic light propagation not supported by higher-symmetry crystals $^{7,8,26-28}$. Here we show exemplary consequences of these material features for nanophotonics, in particular the emergence of a new form of waves - hyperbolic shear polaritons (HShPs) - which have not been previously observed.
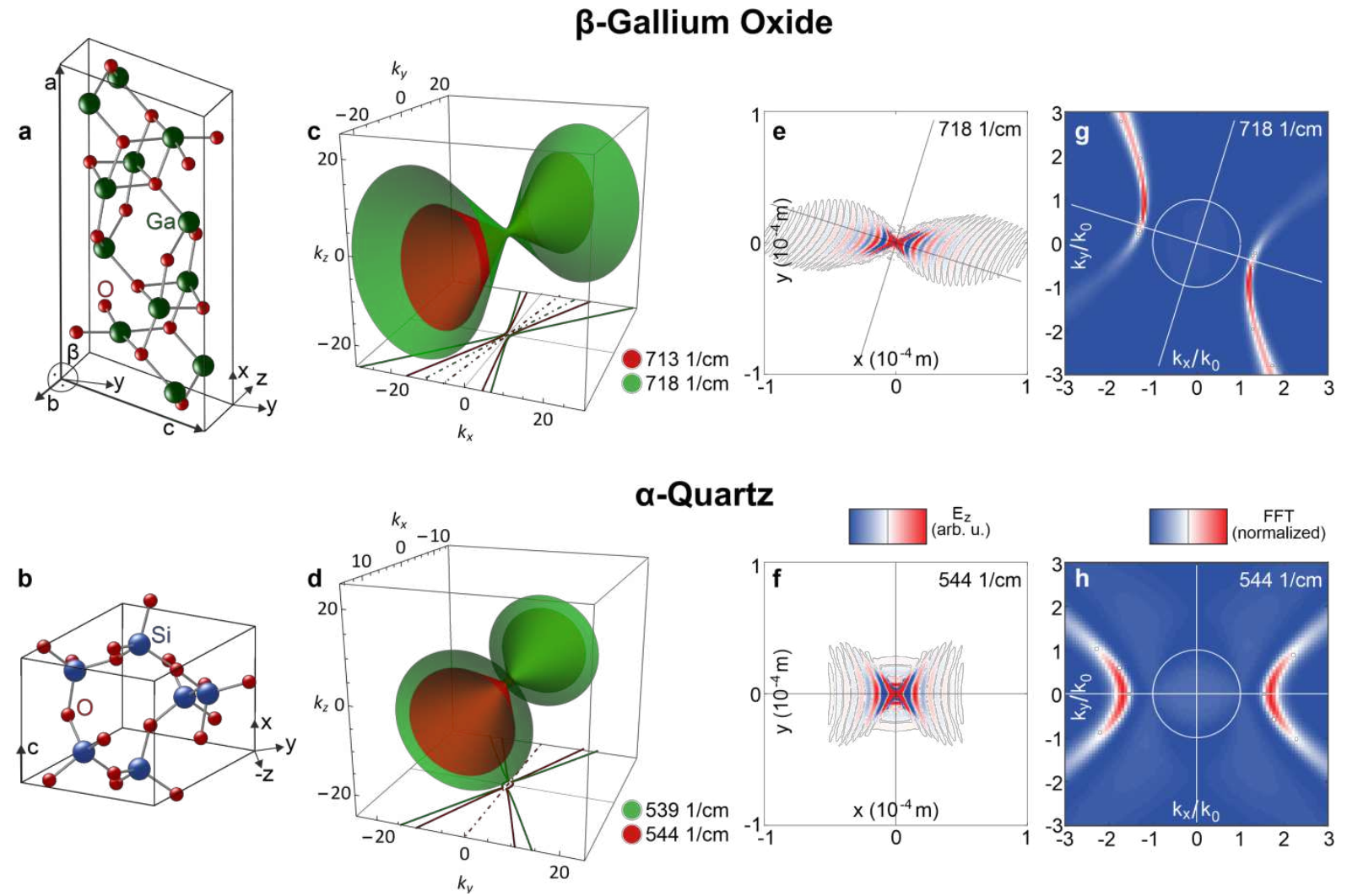

Fig. 1: Hyperbolic shear polaritons in monoclinic $\beta$-gallium oxide (bGO) compared to hyperbolic polaritons in uniaxial $\alpha$-quartz (aQ). (a) Monoclinic crystal structure of bGO (monoclinic angle $\beta=$ $\left.103.7^{\circ}\right)$. The sample surface of the investigated bGO crystal is the monoclinic (010) plane (x-y-plane) (b) Rhombic unit cell of aQ with the c-axis oriented along the $x$-direction, lying in the sample surface plane. The Cartesian coordinate system used in this work is shown. $(c, d)$ Isofrequency surfaces for $b G O$ and $a Q$, respectively, at two frequencies (red and green) where the material supports hyperbolic polaritons. The contour lines at $k_{z}=0$ are plotted as solid lines at the bottom, and their mirror axes are shown as dash-dotted lines. (e,f) Real-space electric fields at the bGO and aQ surface, respectively, and $(g, h)$ the respective two-dimensional Fourier transformation. Lines indicate the frequency dependent optical axes for bGO and the crystal axes for aQ. The fields were calculated using COMSOL Multiphysics ${ }^{29}$ (see methods for details). 
In this work, we theoretically and experimentally demonstrate the emergence of HShPs in monoclinic crystals. HShPs arise in low-symmetry materials because of the presence of multiple phonon oscillators with non-orthogonal relative orientations. The resulting non-diagonalizable dielectric tensor exhibits shear dissipation terms analogous to viscous flow ${ }^{30}$, which, when strongly coupled to light, enables the emergence of HShPs - a new form of HPs. As an exemplary material to demonstrate this phenomenon, we study beta-phase $\mathrm{Ga}_{2} \mathrm{O}_{3}$ (bGO), which has gained significant research and industrial attention for its high breakdown field ${ }^{31,32}$ and applications in photovoltaics ${ }^{33}$, optical displays $^{34}$ and gas sensors ${ }^{35}$. In the low energy range, bGO features several strong IR-active, nonorthogonal phonon resonances ${ }^{7}$, making the permittivity tensor of bGO naturally non-diagonalizable. Its low-symmetry has two consequences on the polariton propagation when compared to more conventional hyperbolic materials with a diagonal permittivity tensor such as $h B N, a Q$, and $\alpha-M_{o} O_{3}$. First, both the bGO polariton wavelength and propagation direction strongly disperse with frequency. Second, we demonstrate that the asymmetric nature of optical loss in such crystals gives rise to shear, resulting in polariton propagation with tilted wave fronts. Such tilted wave fronts are a direct consequence of the material's low symmetry and are one of the most significant and unique features of HShPs. New opportunities for polaritonics arise for HShPs stemming directly from their nonHermitian and topological nature. Yet, quite surprisingly, they can be observed in low-loss, naturally occurring materials, without the need for artificial structuring of a material surface ${ }^{23}$.

To highlight the role of the asymmetry of monoclinic crystals in their polariton response, we compare HShPs with HPs supported by higher-symmetry anisotropic crystals, such as $\alpha$-Quartz $(\mathrm{aQ})^{36}$. In this vein, we compare the crystal structure of monoclinic bGO in Fig 1a with the one of trigonal aQ in Fig. $1 b$, illustrating the non-perpendicular crystal axes present in bGO. In general, the description of the dielectric response of monoclinic crystals requires inclusion of off-diagonal elements in the monoclinic plane within the frequency-dependent, complex-valued dielectric tensor $\overline{\overline{\varepsilon(\omega)}}$

$$
\overline{\overline{\varepsilon(\omega)}}=\left[\begin{array}{ccc}
\varepsilon_{x x}(\omega) & \varepsilon_{x y}(\omega) & 0 \\
\varepsilon_{x y}(\omega) & \varepsilon_{y y}(\omega) & 0 \\
0 & 0 & \varepsilon_{z z}(\omega)
\end{array}\right] .
$$

Due to reciprocity, the off-diagonal elements are identical. The coordinate systems employed to define the response of $b G O$ and $a Q$ are sketched in Fig. 1a and b, respectively. To analyze the properties of HShPs in monoclinic materials, we first rigorously solve Maxwell's equations (see methods) to calculate the dispersion relation of the polaritonic modes supported by bGO and - for comparison $-\mathrm{aQ}$, each at two distinct frequencies. Initially, we consider the lossless case, in which the imaginary part of each term in the dielectric tensor is neglected for both bGO and aQ. The solutions for the polariton wavevectors in both materials at two different frequencies are provided in Fig. $1 c$ and d. For aQ, we observe two open hyperboloid surfaces - as expected for uniaxial hyperbolic materials - where a change in frequency results in a corresponding change in wavevector, while preserving the hyperboloid orientation, i.e., the direction of polariton propagation (Fig. $1 \mathrm{~d}$ ). In contrast, as we change the frequency, not only the bGO polariton wavevector magnitude changes, but also the direction of the hyperboloid rotates within the monoclinic plane, as can be appreciated by examining the $k_{z}=0$ projections (Fig. $1 \mathrm{c}$, bottom). This is a direct consequence of the non-trivial relative orientation of the phonon resonances supporting the hyperbolic response ${ }^{7}$, which results in polariton bands that disperse in azimuth angle as a function of frequency. This feature represents a signature of reduced symmetry associated with HShPs supported in monoclinic crystals (and is also anticipated in triclinic crystals), in contrast to HPs observed in higher symmetry crystals.

When we also account for natural material losses resulting from inherent phonon scattering processes, the polariton propagation in bGO displays a reduced symmetry in comparison to 
hyperbolic polaritons in aQ even at individual frequencies, as illustrated in Fig. $1 \mathrm{e}$ and $\mathrm{f}$. In these panels, we show the results of full-wave calculations of light propagating across the surface of a semi-infinite slab of bGO and $y$-cut $a Q$, where in both cases natural material losses were explicitly taken into account. The surface waves are launched by a point dipole placed just above the material surface. For in-plane hyperbolic materials, these surface waves are referred to as hyperbolic surface polaritons or hyperbolic Dyakonov polaritons ${ }^{37,38}$, and constitute a subset of HPs supported in these materials similar to volume-confined HPs in thin films. At an isotropic-hyperbolic material interface, HPs display a hyperbolic dispersion within the surface plane, and have been analytically studied for uniaxial and biaxial materials previously ${ }^{38-41}$. For aQ, HPs spread out along one crystal axis of the surface, and are symmetric with respect to the crystal axes, as it can be confirmed by a Fourier transform of the real-space electric field profile (Fig. 1h). However, for bGO in Fig. 1e we observe that HPs are rotated with respect to the coordinate system of the monoclinic plane, as anticipated by the isofrequency contours (Fig. 1c). In addition, the wavefronts are tilted with respect to the direction of energy flow, with no apparent mirror symmetry. This feature can also be clearly seen by examining the Fourier transform of the real-space profile (Fig. 1g), exhibiting a stronger intensity along one side of the hyperbola. These observations constitute the discovery of HShPs in low-symmetry crystals.
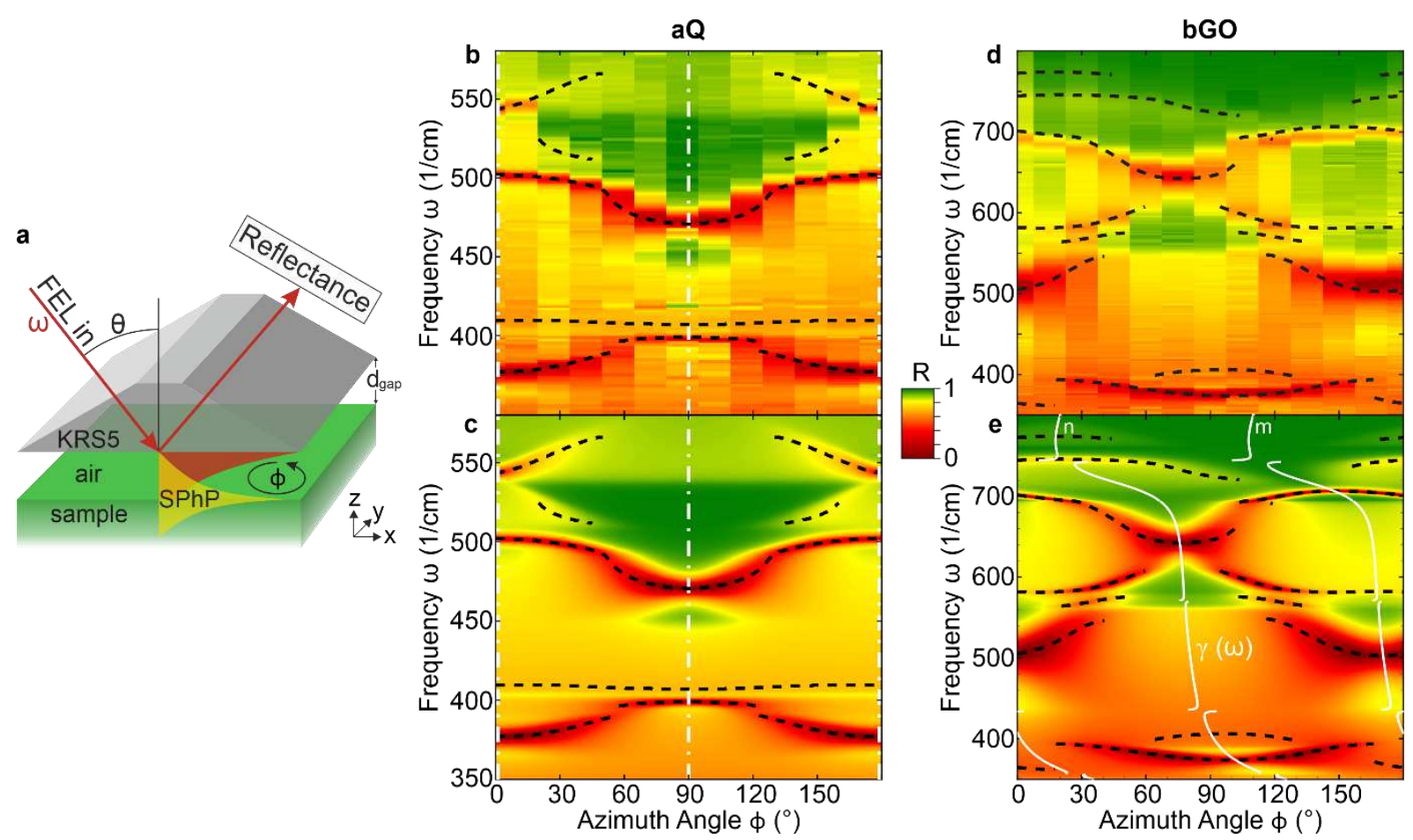

Fig. 2 Experimental observation of HPs on aQ and HShPs on bGO. (a) Otto-type prism coupling configuration for the experimental observation of surface waves. The p-polarized free-electron laser (FEL) excitation beam is reflected at the prism back side at an incident angle of $\boldsymbol{\theta}=\mathbf{2 8}^{\circ}$. The reflectance detection is unpolarized. The gap size was fixed to $\boldsymbol{d}_{\text {gap }} \approx \mathbf{1 0 . 4} \boldsymbol{\mu m}$ for $a Q$, and $\boldsymbol{d}_{\text {gap }} \approx$ 8. $3 \mathrm{\mu m}$ for bGO. Data sets at other gap sizes are shown in Supplementary Fig. S3. (b) Experimental azimuth dependence of $H P s$ on $a Q$, and (c) corresponding simulated reflectance map calculated by means of a transfer matrix method ${ }^{42}$. (d) Experimental azimuth dependence of HShPs on bGO, and (e) corresponding simulated reflectance map. The white lines in (e) correspond to the directions of the frequency-dependent major polarizability axes $n$ and $m$, see text.

In order to experimentally demonstrate the effects of reduced symmetry in polariton propagation in bGO in contrast to higher-symmetry materials, we compare the dispersion of HShPs in bGO to the one of HPs in aQ using an Otto-type prism coupling geometry ${ }^{43,44}$ (sketched in Fig. 2a, for details see methods). This method measures the spectral dependence of surface waves through sharp 
absorption peaks observed as dips in the reflectance spectra, by using a prism placed near the material surface. The crystals are oriented such that the monoclinic plane (bGO) and the optical axis $(\mathrm{aQ})$ are parallel to the sample surface. By following the spectral position of the polariton resonances as a function of azimuth angle, we probe the dispersion of hyperbolic waves at the surface for both bGO and aQ. The Otto geometry effectively selects a specific in-plane momentum component of those surface waves induced by the dipole excitation in Fig 1e and $f$, as set by the incidence angle $\theta$ and azimuth angle $\Phi$ that define the magnitude ${ }^{43,44}$ and direction of the selected momentum, respectively. The retrieved experimental dispersion of HPs on the surface of $a Q$ is shown in Fig. $2 b$, in excellent agreement with the corresponding simulations (Fig. 2c). The dips in the measured reflectance spectra reveal the supported polariton modes, which are only observable along specific azimuth angles and are symmetric about the crystal axes, $\phi=0^{\circ}\left(180^{\circ}\right)$ and $90^{\circ}$. In contrast, the experimentally measured azimuthal dependence of HShPs on monoclinic bGO (Fig. 2d), in excellent agreement with the simulated dispersion curves (Fig. 2e), exhibits no mirror symmetry. The reduced symmetry observed within the polaritonic dispersion is a direct consequence of the lack of symmetry in the vibrational structure of $\mathrm{bGO}^{7}$. As a consequence, the HShPs are not propagating along fixed axes, but display a continuous rotation of the propagation direction as the frequency is varied.

To describe the nature of the rotation of the HShP propagation direction for bGO, we diagonalize the real part of the permittivity tensor individually at each frequency. Since $\operatorname{Re}[\overline{\varepsilon(\omega)}]$ is Hermitian, we can diagonalize it, following the Spectral Theorem, by rotating the monoclinic plane by the frequency-dependent angle

$$
\gamma(\omega)=\frac{1}{2} \arctan \left(\frac{2 \operatorname{Re}\left(\varepsilon_{\mathrm{xy}}\right)(\omega)}{\operatorname{Re}\left(\varepsilon_{\mathrm{xx}}\right)(\omega)-\operatorname{Re}\left(\varepsilon_{\mathrm{yy}}\right)(\omega)}\right) .
$$

The dispersion of $\gamma(\omega)$ is shown in Fig $2 \mathrm{e}$ (white lines), illustrating that the major polarizability directions within the monoclinic plane, denoted as $m$ and $n$, widely vary across the spectrum. This frequency-dependent coordinate system enables an easier understanding of the polaritonic response, in particular a straightforward classification of dielectric, elliptical, as well as type I and II hyperbolic frequency ranges for the polariton dispersion (see SI section 1 for details). The rotated coordinate axes are shown in Fig. 1e and g, illustrating their alignment with the hyperbolic dispersion.

Whilst the frequency variation of the propagation direction can be described by the coordinate rotation in Eq. 2, such rotation does not capture the tilted wave fronts observed in Fig 1e. This is because, as we choose the rotated coordinate system [mnz], we still retain a purely imaginary offdiagonal permittivity component (see SI section 1 for details). These terms are associated with the non-orthogonal relative orientation of the material resonances, coupling the two crystal axes in the monoclinic plane. As a result, even in the rotated coordinate system [mnz] the dielectric tensor has off-diagonal terms associated to shear dissipation, linking different propagation directions purely through light absorption, analogous to the physics of viscous fluid flow ${ }^{30}$. 

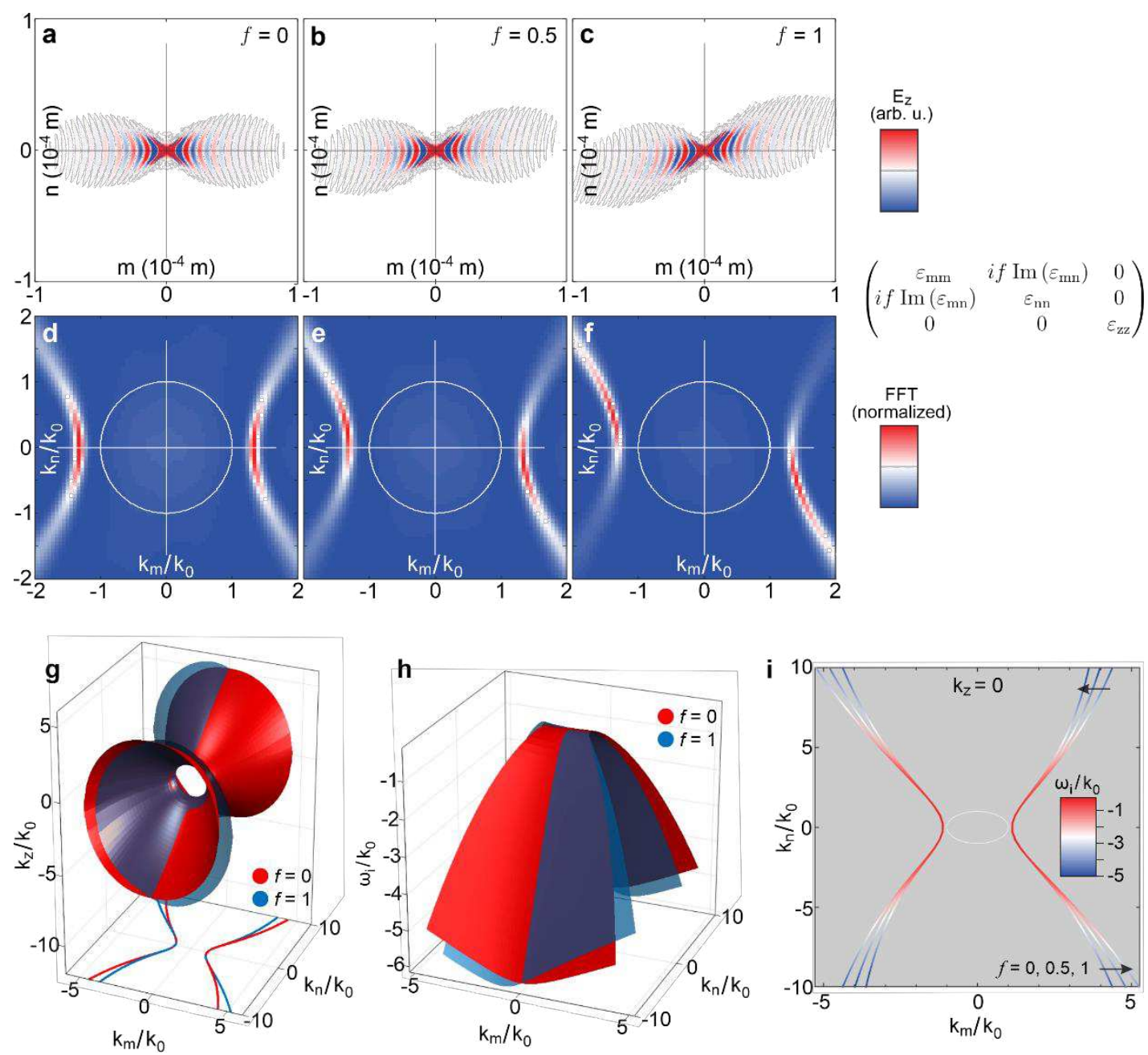

Fig. 3: Symmetry breaking by shear dissipation for hyperbolic polaritons in monoclinic bGO. (a-c) Real-space electric fields at the bGO surface for three different magnitudes of the off-diagonal permittivity element if $\boldsymbol{I} \operatorname{Im}\left(\boldsymbol{\varepsilon}_{\boldsymbol{m} n}\right)$ calculated in the frequency-dispersive rotated coordinate system at $718 \mathrm{~cm}^{-1}$, and $(d-f)$ respective Fourier transformation $k$-space maps in arbitrary units. The thin crosshairs indicate the principal axes in the frequency-dispersive rotated coordinate system, aligned horizontally and vertically because the maps were calculated within the rotated coordinate system [mnz]. (g) Isofrequency surface of bulk eigenmodes in bGO with complex frequency $\widetilde{\boldsymbol{\omega}}=\boldsymbol{\omega} / \boldsymbol{k}_{\mathbf{0}}=\mathbf{1}+$ $\boldsymbol{i} \boldsymbol{\omega}_{\boldsymbol{i}} / \boldsymbol{k}_{\mathbf{0}}$ calculated in the rotated frame for $\boldsymbol{f}=\mathbf{0}, \mathbf{1}$ (red and blue, respectively). See methods for details on the approach. (h) Imaginary part $\boldsymbol{\omega}_{\boldsymbol{i}}$ for $\boldsymbol{f}=\mathbf{0}, \mathbf{1}$. (i) Contour lines of the isofrequency surface at $\boldsymbol{k}_{\mathbf{z}}=\mathbf{0}$ for $\boldsymbol{f}=\mathbf{0}, \mathbf{0 . 5}, \mathbf{1}$. The imaginary part $\boldsymbol{\omega}_{\boldsymbol{i}}$ at the corresponding point in $k$-space is color-coded.

To selectively probe the role of shear dissipation, we simulate the polariton propagation in the rotated coordinate system [mnz] at $7181 / \mathrm{cm}$. In order to highlight the role of shear, we include an arbitrary scaling factor for the magnitude of the off-diagonal imaginary component, indicated as $i *$ $f \operatorname{Im}\left(\varepsilon_{\mathrm{mn}}\right)$, with $f=0,0.5$, and 1 (shown in Fig. 3a-c), while retaining the diagonal loss terms. When we remove the off-diagonal component $(f=0)$, bGO essentially becomes a shear-free biaxial material, akin to $\mathrm{MoO}_{3}$ and similar to uniaxial aQ, with polaritons propagating along the optical axes (Fig. 3a). Thus, while polariton propagation in bGO is anisotropic in specific spectral ranges (similar to polaritons in $\mathrm{MoO}_{3}{ }^{3,22}$ ), mode propagation without shear dissipation is symmetric about the (frequency-dependent) major polarizability axes (Fig. 3a). As we gradually increase the magnitude of the off-diagonal loss term back to its natural value $(f=1)$, the wavefronts become increasingly skewed from the major polarizability axis (Fig. 3b,c). This behavior cannot be expected in higher- 
symmetry materials where polariton propagation is observed only along the principal crystal axes $^{3,4,22}$. To extract the polariton dispersion from the real-space profiles (Fig. $3 \mathrm{a}-\mathrm{c}$ ), we perform a 2D Fourier transformation for each of the modes, revealing strong symmetry breaking in the intensity distribution within the isofrequency hyperbolae observed in the reciprocal space maps in Fig. 3d-f. This observation provides further evidence that the propagation of polaritons is non-trivial within low-symmetry monoclinic, and by extension triclinic, systems.

In order to connect the reduced symmetry of the surface subset of HShPs observed here experimentally (Fig. 2) and through our simulations (Fig. 3a-c) to the more general HShPs in the bulk, we now calculate isofrequency surfaces for polariton modes in bGO explicitly including loss, in order to account for the effect of shear dissipation. To this end, we solve Maxwell's equations for real momentum values, yielding complex frequency eigenvalues, whose imaginary part accounts for finite lifetime of the supported modes (see Methods for details). The results of these calculations are shown in Fig. 3g-i. The real part of the eigen-frequency is fixed here, and we find its imaginary part $\omega_{i}$ (Fig. 3h) and the corresponding value of $k_{z}$ (Fig. 3g) for each pair of $k_{m}$ and $k_{n}$. We note that $\omega_{i}$ is proportional to the inverse of the modal lifetime $\tau \sim 2 \pi / \omega_{i} c$, where $c$ is the speed of light speed in vacuum. The lifetime in our example at $718 \mathrm{~cm}^{-1}$ is estimated as $0.22 \mathrm{ps}$ near $k_{n}=0$, consistent with experimental records in the literature ${ }^{45}$. The calculations are performed in the rotated coordinate system for both $f=0$ and $f=1$, showing that both the shape of the isofrequency surfaces, as well as their lifetimes, change significantly with the inclusion of the shear dissipation term in the off-diagonal imaginary components. Notably, these calculations prove that at individual frequencies and in the major polarizability frame, mirror symmetry of polariton propagation is lost in monoclinic materials as a direct consequence of the shear dissipation.

To relate the isofrequency contours of HShPs to the surface mode dispersions in Fig. $3 \mathrm{~d}-\mathrm{f}$, we plot the $k_{z}=0$ solution in Fig. $3 i$ with the color scale indicating the relative loss $\omega_{i}$ of the mode. Two important observations can be made: first, the mirror symmetry of the isofrequency curves is broken for $f>0$ and it requires higher-order terms to account for the asymmetric shape; second, the mode losses are redistributed asymmetrically, with losses decreasing in one arm of the hyperbolae, while increasing on the other arm. This redistribution of loss is in excellent qualitative agreement with the point dipole simulations presented in Fig. 1 and suggests that the reduced/increased loss within the two arms of the hyperbolae contributes to the asymmetry in the intensity distributions in Fig. 3d-f. Interestingly, in this example we also observe an increase in propagation lengths as we increase $f$, despite the fact that the loss in the diagonal terms are kept constant. In this scenario, the additional shear dissipation induces an effective reduction of propagation losses (see SI Section 5 for details), associated with the tilting of the eigen-vectors.

These observations naturally link HShPs in monoclinic crystals to the rich emerging area of nonHermitian and topological photonics. While loss in orthogonal systems alone can already have interesting consequences for polariton propagation ${ }^{46}$, the off-diagonal shear dissipation highlighted here can provide new opportunities for non-Hermitian photonics and for manipulation of topological polaritons in low-symmetry materials. For instance, we envision asymmetric topological transitions experienced by HShPs, generalizing previous results in orthorhombic systems ${ }^{2}$ by exploiting the unique non-Hermitian features emerging in low-symmetry materials. In addition, there have been recent studies suggesting the connection between Dyakonov surface waves and surface states emerging from one-dimensional bands degeneracy (nodal lines) of topological nature of highsymmetry metacrystals ${ }^{47}$. We anticipate that HShPs may generalize these opportunities to asymmetric topological bands in which non-Hermiticity in the natural materials plays a dominant role. 
In this paper we have demonstrated that low-symmetry crystals can support a new class of hyperbolic polariton modes with their symmetry broken by shear phenomena, which we refer to as HShPs. We introduce bGO as an exemplary material to enable these phenomena, and experimentally demonstrate the symmetry-broken dispersion of the supported surface waves. The nondiagonalizable dielectric permittivity plays a key role in the unique properties of low-symmetry crystals including monoclinic and triclinic lattices. Our results are generalizable to engineered photonic systems with at least two non-orthogonal oscillators, including new metasurface designs capturing these physics. We anticipate that HShPs may have important implications in the manipulation of phase and directional energy transfer, including radiative heat transport ${ }^{48,49}$, ultrafast asymmetric thermal dissipation in the near-field ${ }^{49}$, and gate-tunability for on-chip all-optical circuitry ${ }^{50,51}$. Beyond nanophotonics advances, infrared polariton propagation has been demonstrated as a means for quantifying crystal strain ${ }^{52-54}$, polytypes ${ }^{55,56}$, variations in free-carrier density, as well as phononic and electronic properties around defects ${ }^{54}$ with nanoscale precision (< $20 \mathrm{~nm}$ ), thereby promising a novel metrology tool in characterizing low-symmetry ultra-wide bandgap semiconductors. We highlight that our results are applicable to any material with non-orthogonal optically active transitions, and therefore may be extended to other optical phenomena, such as excitons in triclinic $\mathrm{ReSe}_{2}$, which have recently been shown to support multiple in-plane selective excitons ${ }^{57}$.

Beyond the results provided here for intrinsic, undoped bGO, we also note that the presence of free charge carriers in $\mathrm{bGO}^{58}$ - introduced either by impurity doping or transiently through photo doping - may allow for methods to modulate the major polarizability axes, enabling direct steering of the HShP propagation direction (see SI Section 4). Finally, exfoliation of thin flakes of single-crystal bGO has also been recently reported ${ }^{59}$. Making use of volume-confined HShPs in bGO will enable all the functionalities of uniaxial/biaxial hyperbolic systems, with the additional features unveiled by shear dissipation. As such, HShPs supported in low-symmetry semiconductors offer a broad scope for uncovering and exploiting new types of light-matter waves and utilizing their properties in the midto far-infrared for an extensive range of applications, including on-chip photonics, light focusing, beam steering, and controlling thermal emissivity and radiation. 


\section{Methods}

\section{Experimental}

The insulating bGO sample was produced via Fe compensation doping and was purchased from Novel Crystal Technology, Inc., Japan. The aQ sample was purchased from MaTeck GmbH, Germany. The absolute azimuth orientation of the samples was extracted from a global fit for each of the data sets of $\mathrm{aQ}$ and bGO (plotted in Fig. $1 \mathrm{a}$ and e, respectively), resulting in a rotation with respect to the principal $x$-axis of the laboratory coordinate system of $\Delta \phi_{\mathrm{bGO}}=27.95^{\circ}$ and $\Delta \phi_{\mathrm{aQ}}=26.96^{\circ}$. The aQ data has been rotated accordingly in order to shift the crystal axes (mirror planes) onto multiples of $90^{\circ}$. On the other hand, the bGO data is plotted as measured since there is no principal azimuth angle for alignment because of the broken mirror symmetry. Here, the simulation was rotated accordingly to match the data.

As an excitation source for the Otto-type prism coupled experiments, we employ a mid-IR free electron laser (FEL) with small bandwidth $(\sim 0.3 \%)$ and wide tunability of $3-50 \mu \mathrm{m}$, covering the spectral ranges $(350-8001 / \mathrm{cm})$ where $\mathrm{aQ}$ and bGO support polaritonic modes (details on the FEL have been reported elsewhere ${ }^{60}$ ). While the frequency is scanned by tuning the FEL, different inplane momenta can be accessed via changes in the incidence angle $\theta$ by rotating the entire Otto geometry (details on the setup have been reported elsewhere ${ }^{43,61}$ ). For the conducted experiments, the incident angle was fixed to $28^{\circ}$, resulting in an in-plane momentum of $k_{\|} / k_{0} \approx 1.10$ (at $5001 / \mathrm{cm})$. In contrast to alternative approaches, the Otto geometry features experimental control over the excitation efficiency through tunability of the air gap width $d_{\text {gap }}$. Here, the gap was adjusted to a separation where all excited modes could be observed in the spectra simultaneously, that is, $d_{\text {gap }} \approx 8.3 \mu \mathrm{m}$ for $\mathrm{bGO}$, and $d_{\text {gap }} \approx 10.4 \mu \mathrm{m}$ for aQ. Direct read-out of $d_{\text {gap }}$ with a range of $1-$ $50 \mu \mathrm{m}$ is realized via whitelight interferometry, while the contrast of the interference spectrum grants parallel alignment of prism and sample ${ }^{61}$.

\section{Theoretical}

\section{Transfer Matrix}

The calculations of the optical response shown in Fig. 2c,e and the dispersion maps in Fig. S2c,d were performed using a generalized $4 \times 4$ transfer matrix formalism ${ }^{42}$. In short, the formalism allows for the calculation of reflection and transmission coefficients in any number of stratified media with arbitrary dielectric tensor, which allows to account for the anisotropy of our samples.

\section{COMSOL Simulations}

COMSOL version 5.6 was used for simulating point dipole excitation of HShPs on bGO. A point dipole was placed $100 \mathrm{~nm}$ above the surface of an infinite slab of bGO, with a dielectric permittivity matching that of Ref. 7. The dielectric function of aQ was taken from Ref. ${ }^{62}$. Perfectly matched impedance boundary conditions were used on the sides of the simulation, which in principle absorb all radiation. However, to account for the imperfect behavior of the boundaries we ensured that the bGO slab was sufficiently large ( 250 by 250 by $8 \mu \mathrm{m}$ ), such that the wave is sufficiently damped when it reaches the boundary as not to influence the results.

\section{Isofrequency Surface with Complex Frequency}

To obtain the isofrequency contour surface of the bulk wave when losses are considered in the materials, we resolve to the complex-frequency method and solve the source-free Maxwell equations as follows,

$$
\left(\begin{array}{ccc}
\widetilde{\omega}^{2} \epsilon_{x x}-\left(\tilde{k}_{z}^{2}+\tilde{k}_{y}^{2}\right) & \widetilde{\omega}^{2} \epsilon_{x y}+\tilde{k}_{x} \tilde{k}_{y} & \tilde{k}_{x} \tilde{k}_{z} \\
\widetilde{\omega}^{2} \epsilon_{x y}+\tilde{k}_{x} \tilde{k}_{y} & \widetilde{\omega}^{2} \epsilon_{y y}-\left(\tilde{k}_{z}^{2}+\tilde{k}_{x}^{2}\right) & \tilde{k}_{y} \tilde{k}_{z} \\
\tilde{k}_{x} \tilde{k}_{z} & \tilde{k}_{y} \tilde{k}_{z} & \widetilde{\omega}^{2} \epsilon_{z z}-\left(\tilde{k}_{y}^{2}+\tilde{k}_{x}^{2}\right)
\end{array}\right)\left(\begin{array}{l}
E_{x} \\
E_{y} \\
E_{z}
\end{array}\right)=0,
$$


where $\epsilon_{n n}=\epsilon_{n n}^{r}+i \epsilon_{n n}^{k}, n n=x x, y y, z z, \epsilon_{x y}=\epsilon_{x y}^{r}+i \epsilon_{x y}^{k}$. We fix the real component $k_{0}$ of the complex frequency $\omega$ and normalize it as $\widetilde{\omega}=\frac{\omega}{k_{0}}=1+i \widetilde{\omega}_{i}, \widetilde{\omega}^{2}=1-\widetilde{\omega}_{i}^{2}+2 i \widetilde{\omega}_{i}$, where $\omega_{i}$ is proportional to the inverse mode lifetime, and normalize the wavevector as $\tilde{k}_{x, y, z}=\frac{k_{x, y, z}}{k_{0}}$. Note that we choose a negative sign for the time dependent term $e^{-i \omega t}$, so $\widetilde{\omega}_{i}$ must be real and negative to reflect the decay nature of the wave. The analytic expression $F\left(\widetilde{\omega}_{i}, \tilde{k}_{x}, \tilde{k}_{y}, \tilde{k}_{z}\right)=0$ is found via the secular equation of the above matrix, and two equations are obtained by separating the real and imaginary components of $F$, namely,

$$
\begin{aligned}
& F_{r}\left(\widetilde{\omega}_{i}, \tilde{k}_{x}, \tilde{k}_{y}, \tilde{k}_{z}\right)=0, \\
& F_{i}\left(\widetilde{\omega}_{i}, \tilde{k}_{x}, \tilde{k}_{y}, \tilde{k}_{z}\right)=0 .
\end{aligned}
$$

The isofrequency contour of the bulk wave and the imaginary component $\widetilde{\omega}_{i}$ are evaluated from those two equations. The numerical examples at $718 \mathrm{~cm}^{-1}$ are given in Fig. $3(i, j)$ based on the above method $\left(k_{x, y} \rightarrow k_{m, n}\right)$.

When $\tilde{k}_{z}=0$, the analytic expression for the isofrequency contour of bulk wave can be written as

$$
\left[\widetilde{\omega}^{2}\left(\varepsilon_{x x} \varepsilon_{y y}-\varepsilon_{x y}^{2}\right)-\left(\varepsilon_{x x} \tilde{k}_{x}^{2}+\varepsilon_{y y} \tilde{k}_{y}^{2}+2 \varepsilon_{x y} \tilde{k}_{x} \tilde{k}_{y}\right)\right]=0,
$$

which turns into two equations by separating the real and imaginary parts,

$$
\begin{gathered}
\left(1-\widetilde{\omega}_{i}^{2}\right) F_{r}-2 \widetilde{\omega}_{i} F_{k}-\left(\varepsilon_{x x}^{r} \tilde{k}_{x}^{2}+\varepsilon_{y y}^{r} \tilde{k}_{y}^{2}+2 \varepsilon_{x y}^{r} \tilde{k}_{x} \tilde{k}_{y}\right)=0, \\
2 \widetilde{\omega}_{i} F_{r}+\left(1-\widetilde{\omega}_{i}^{2}\right) F_{k}-\left(\varepsilon_{x x}^{k} \widetilde{k}_{x}^{2}+\varepsilon_{y y}^{k} \widetilde{k}_{y}^{2}+2 \varepsilon_{x y}^{k} \widetilde{k}_{x} \widetilde{k}_{y}\right)=0 ; \\
F_{r}=\varepsilon_{x x}^{r} \varepsilon_{y y}^{r}-\varepsilon_{x x}^{k} \varepsilon_{y y}^{k}-\left(\varepsilon_{x y}^{r}{ }^{2}-\varepsilon_{x y}^{k}{ }^{2}\right), \\
F_{k}=\varepsilon_{x x}^{r} \varepsilon_{y y}^{k}+\varepsilon_{x x}^{k} \varepsilon_{y y}^{r}-2 \varepsilon_{x y}^{r} \varepsilon_{x y}^{k} .
\end{gathered}
$$

and they can be further simplified into the following equation

$\left[\left(\varepsilon_{x x}^{r} F_{k}-\varepsilon_{x x}^{k} F_{r}\right) \tilde{k}_{x}^{2}+2\left(\varepsilon_{x y}^{r} F_{k}-\varepsilon_{x y}^{k} F_{r}\right) \tilde{k}_{x} \tilde{k}_{y}+\left(\varepsilon_{y y}^{r} F_{k}-\varepsilon_{y y}^{k} F_{r}\right) \tilde{k}_{y}^{2}\right]^{2}+4\left(F_{r}^{2}+F_{k}^{2}\right)\left[\left(\varepsilon_{x x}^{r} F_{r}+\right.\right.$ $\left.\left.\varepsilon_{x x}^{k} F_{k}\right) \tilde{k}_{x}^{2}+2\left(\varepsilon_{x y}^{r} F_{r}+\varepsilon_{x y}^{k} F_{k}\right) \tilde{k}_{x} \tilde{k}_{y}+\left(\varepsilon_{y y}^{r} F_{r}+\varepsilon_{y y}^{k} F_{k}\right) \tilde{k}_{y}^{2}-F_{r}^{2}-F_{k}^{2}\right]=0$.

Therefore, the isofrequency curves in the $\tilde{k}_{z}=0$ plane as well as the imaginary component of bulk complex frequency are obtained from the above expression. 


\section{Acknowledgements}

The authors wish to thank W. Schöllkopf and S. Gewinner (FHI Berlin) for operating the infrared free-electron laser. NCP, MW, and AP wish to thank Ralph Ernstorfer (FHI Berlin) and Sebastian Mährlein (FHI Berlin) for careful reading of the manuscript. GH acknowledges the support from A*STAR AME Young Individual Research Grants (YIRG, No. A2084c0172). XN, GH, AA acknowledge the Office of Naval Research with grant No. N0001419-1-2011 and the Vannevar Bush Faculty Fellowship. MS acknowledges National Science Foundation awards DMR 1808715, DMR 1420645, and OIA-2044049, and Air Force Office of Scientific Research awards FA9550-181-0360 and FA9550-19-S-0003. JDC was supported by the Office of Naval Research grant \#N00014-18-12107 and JRM by the National Science Foundation, Division of Materials Research grant \#1904793.

\section{Author information}

\section{Affiliations}

Fritz Haber Institute of the Max Planck Society, Berlin, Germany

Nikolai C. Passler, Martin Wolf, Alexander Paarmann

Photonics Initiative, Advanced Science Research Center, City University of New York, New York, NY 10031, USA

Xiang Ni, Guangwei Hu, Andrea Alù

Department of Electrical and Computer Engineering, National University of Singapore, Singapore

Guangwei Hu

Vanderbilt University, Nashville, TN, USA

Joseph R. Matson, Joshua D. Caldwell

University of Nebraska, Lincoln, NE, USA

Mathias Schubert

The University of lowa, lowa City, IA, USA

Thomas G. Folland

Corresponding author

Correspondence to Andrea Alù, Joshua D. Caldwell, Thomas G. Folland, and Alexander Paarmann.

\section{Contributions}

NCP performed the experiments. NCP and AP performed the transfer matrix simulations. NCP, TGF, and $\mathrm{AP}$ performed $\mathrm{COMSOL}$ simulations. $\mathrm{XN}, \mathrm{GH}$, and $\mathrm{AA}$ performed the analytical derivations and 
the calculation of isofrequency surfaces. All authors contributed to writing the manuscript. AA, JDC, TFG, and AP oversaw the project. MS and JDC initiated the research. 


\section{References}

1. Ma, W. et al. In-plane anisotropic and ultra-low-loss polaritons in a natural van der Waals crystal. Nature 562, 557-562 (2018).

2. $\mathrm{Hu}, \mathrm{G}$. et al. Topological polaritons and photonic magic angles in twisted $\alpha-\mathrm{MoO} 3$ bilayers. Nature 582, 209-213 (2020).

3. Zheng, Z. et al. A mid-infrared biaxial hyperbolic van der Waals crystal. Sci. Adv. 5, eaav8690 (2019).

4. Taboada-Gutiérrez, J. et al. Broad spectral tuning of ultra-low-loss polaritons in a van der Waals crystal by intercalation. Nat. Mater. 19, 964-968 (2020).

5. Low, T. et al. Polaritons in layered two-dimensional materials. Nat. Mater. 16, 182-194 (2016).

6. Basov, D. N., Fogler, M. M. \& Garcia de Abajo, F. J. Polaritons in van der Waals materials. Science (80-. ). 354, aag1992-aag1992 (2016).

7. Schubert, M. et al. Anisotropy, phonon modes, and free charge carrier parameters in monoclinic $\beta$-gallium oxide single crystals. Phys. Rev. B 93, 125209 (2016).

8. Claus, R. Polariton Dispersion and Crystal Optics in Monoclinic Materials. Phys. Status Solidi 88, 683-688 (1978).

9. Jacob, Z. Hyperbolic phonon-polaritons. Nat. Mater. 13, 1081-1083 (2014).

10. Dai, S. et al. Tunable Phonon Polaritons in Atomically Thin van der Waals Crystals of Boron Nitride. Science (80-. ). 343, 1125-1129 (2014).

11. Caldwell, J. D. et al. Sub-diffractional volume-confined polaritons in the natural hyperbolic material hexagonal boron nitride. Nat. Commun. 5, 5221 (2014).

12. Armbruster, T. et al. Recommended nomenclature of epidote-group minerals. Eur. J. Mineral. 18, (2006).

13. Liu, M. et al. Symmetry breaking and geometric confinement in VO2: Results from a threedimensional infrared nano-imaging. Appl. Phys. Lett. 104, 121905 (2014).

14. PHILLIPPI, C. M. \& MAZDIYASNI, K. S. Infrared and Raman Spectra of Zirconia Polymorphs. J. Am. Ceram. Soc. 54, (1971).

15. Koch, E. E., Otto, A. \& Kliewwer, K. L. Reflection spectroscopy on monoclinic crystals. Chem. Phys. 3, 362-369 (1974).

16. Basov, D. N., Averitt, R. D. \& Hsieh, D. Towards properties on demand in quantum materials. Nat. Mater. 16, 1077-1088 (2017).

17. Chen, C. W., Choe, J. \& Morosan, E. Charge density waves in strongly correlated electron systems. Reports Prog. Phys. 79, 084505 (2016).

18. Shiozaki, K. \& Sato, M. Topology of crystalline insulators and superconductors. Phys. Rev. B 90, 165114 (2014).

19. Caldwell, J. D. et al. Low-loss, infrared and terahertz nanophotonics using surface phonon polaritons. Nanophotonics 4, 44-68 (2015).

20. Foteinopoulou, S., Devarapu, G. C. R., Subramania, G. S., Krishna, S. \& Wasserman, D. Phononpolaritonics: enabling powerful capabilities for infrared photonics. Nanophotonics 8, 2129- 
2175 (2019).

21. Poddubny, A., lorsh, I., Belov, P. \& Kivshar, Y. Hyperbolic metamaterials. Nat. Photonics 7, 948-957 (2013).

22. Ma, W. et al. In-plane anisotropic and ultra-low-loss polaritons in a natural van der Waals crystal. Nature 562, 557-562 (2018).

23. Li, P. et al. Infrared hyperbolic metasurface based on nanostructured van der Waals materials. Science (80-. ). 359, 892-896 (2018).

24. Duan, J. et al. Twisted Nano-Optics: Manipulating Light at the Nanoscale with Twisted Phonon Polaritonic Slabs. Nano Lett. 20, 5323-5329 (2020).

25. Peck, D. \& Ostrander, A. Crystallography: The Monoclinic System. https://www.mindat.org/article.php/2787/Crystallography\%3A+The+Monoclinic+System (2020). accessed: May 252021

26. Jellison, G. E. et al. Spectroscopic dielectric tensor of monoclinic crystals: CdWO4. Phys. Rev. B 84, 195439 (2011).

27. Sturm, C. et al. Dipole analysis of the dielectric function of color dispersive materials: Application to monoclinic Ga2O3. Phys. Rev. B 94, 035148 (2016).

28. Mock, A. et al. Band-to-band transitions, selection rules, effective mass, and excitonic contributions in monoclinic $\beta-G a 2 O 3$. Phys. Rev. B 96, 245205 (2017).

29. Comsol. COMSOL Multiphysics. accessed: July 27, 2020.

30. Wu, Y., Zhang, W., Wang, Y., Zou, Z. \& Chen, J. Energy dissipation analysis based on velocity gradient tensor decomposition. Phys. Fluids 32, 035114 (2020).

31. Higashiwaki, M. \& Jessen, G. H. Guest Editorial: The dawn of gallium oxide microelectronics. Appl. Phys. Lett. 112, 60401 (2018).

32. Tadjer, M. J. Cheap Ultra-Wide Bandgap Power Electronics? Gallium Oxide May Hold the Answer. Electrochem. Soc. Interface 27, 49-52 (2018).

33. Granqvist, C. G. Handbook of Inorganic Electrochromic Materials. (Elsevier, 1995).

34. Miyata, T., Nakatani, T. \& Minami, T. Gallium oxide as host material for multicolor emitting phosphors. J. Lumin. 87-89, 1183-1185 (2000).

35. Réti, F., Fleischer, M., Meixner, H. \& Giber, J. Effect of coadsorption of reducing gases on the conductivity of $\beta-G a 2 O 3$ thin films in the presence of O2. Sensors Actuators B Chem. 19, 573577 (1994).

36. Winta, C. J., Wolf, M. \& Paarmann, A. Low-temperature infrared dielectric function of hyperbolic $\alpha$-quartz. Phys. Rev. B 99, 144308 (2019).

37. Li, P. et al. Optical Nanoimaging of Hyperbolic Surface Polaritons at the Edges of van der Waals Materials. Nano Lett. 17, 228-235 (2017).

38. Álvarez-Pérez, G., Voronin, K. V., Volkov, V. S., Alonso-González, P. \& Nikitin, A. Y. Analytical approximations for the dispersion of electromagnetic modes in slabs of biaxial crystals. Phys. Rev. B 100, 1-11 (2019).

39. Jacob, Z. \& Narimanov, E. E. Optical hyperspace for plasmons: Dyakonov states in metamaterials. Appl. Phys. Lett. 93, (2008). 
40. Zapata-Rodríguez, C. J., Miret, J. J., Vuković, S. \& Belić, M. R. Engineered surface waves in hyperbolic metamaterials. Opt. Express 21, 19113 (2013).

41. Sorni, J. A., Naserpour, M., Zapata-Rodríguez, C. J. \& Miret, J. J. Dyakonov surface waves in lossy metamaterials. Opt. Commun. 355, 251-255 (2015).

42. Passler, N. C. \& Paarmann, A. Generalized $4 \times 4$ matrix formalism for light propagation in anisotropic stratified media: study of surface phonon polaritons in polar dielectric heterostructures. J. Opt. Soc. Am. B 34, 2128 (2017).

43. Passler, N. C. et al. Second-Harmonic Generation from Critically Coupled Surface Phonon Polaritons. ACS Photonics 4, 1048-1053 (2017).

44. Folland, T. G., Nordin, L., Wasserman, D. \& Caldwell, J. D. Probing polaritons in the mid- to farinfrared. J. Appl. Phys. 125, 191102 (2019).

45. Yoxall, E. et al. Direct observation of ultraslow hyperbolic polariton propagation with negative phase velocity. Nat. Photonics 9, 674-678 (2015).

46. Antoniou, P., Suchanek, F., Varner, J. F. \& Foley, J. J. Role of Cavity Losses on Nonadiabatic Couplings and Dynamics in Polaritonic Chemistry. J. Phys. Chem. Lett. (2020) doi:10.1021/acs.jpclett.0c02406.

47. Gao, W. et al. Experimental observation of photonic nodal line degeneracies in metacrystals. Nat. Commun. 9, (2018).

48. Tielrooij, K.-J. et al. Out-of-plane heat transfer in van der Waals stacks through electronhyperbolic phonon coupling. Nat. Nanotechnol. 13, 41-46 (2018).

49. Salihoglu, H. et al. Energy Transport by Radiation in Hyperbolic Material Comparable to Conduction. Adv. Funct. Mater. 30, 1905830 (2020).

50. Maia, F. C. B. et al. Anisotropic Flow Control and Gate Modulation of Hybrid PhononPolaritons. Nano Lett. 19, 708-715 (2019).

51. Dai, S. et al. Graphene on hexagonal boron nitride as a tunable hyperbolic metamaterial. Nat. Nanotechnol. (2015) doi:10.1038/nnano.2015.131.

52. Bensmann, S. et al. Near-field imaging and spectroscopy of locally strained GaN using an IR broadband laser. Opt. Express 22, 22369 (2014).

53. Gigler, A. M. et al. Nanoscale residual stress-field mapping around nanoindents in SiC by IR sSNOM and confocal Raman microscopy. Opt. Express 17, 22351 (2009).

54. Hauer, B. et al. Exploiting Phonon-Resonant Near-Field Interaction for the Nanoscale Investigation of Extended Defects. Adv. Funct. Mater. 30, 1907357 (2020).

55. Huber, A., Ocelic, N., Taubner, T. \& Hillenbrand, R. Nanoscale Resolved Infrared Probing of Crystal Structure and of Plasmon-Phonon Coupling. Nano Lett. 6, 774-778 (2006).

56. Ocelic, N., Huber, A. \& Hillenbrand, R. Pseudoheterodyne detection for background-free nearfield spectroscopy. Appl. Phys. Lett. 89, 101124 (2006).

57. Arora, A. et al. Highly Anisotropic in-Plane Excitons in Atomically Thin and Bulklike $1 T^{\prime}-$ ReSe2. Nano Lett. 17, (2017).

58. Schubert, M. et al. Longitudinal phonon plasmon mode coupling in $\beta-G a 2 O 3$. Appl. Phys. Lett. 114, 102102 (2019).

59. Hwang, W. S. et al. High-voltage field effect transistors with wide-bandgap $\beta$-Ga 203 
nanomembranes. Appl. Phys. Lett. 104, (2014).

60. Schöllkopf, W. et al. The new IR and THz FEL facility at the Fritz Haber Institute in Berlin. Proc. SPIE 9512, 95121L (2015).

61. Pufahl, K. et al. Controlling nanoscale air-gaps for critically coupled surface polaritons by means of non-invasive white-light interferometry. Appl. Phys. Lett. 113, 161103 (2018).

62. Spitzer, W. G. \& Kleinman, D. A. Infrared Lattice Bands of Quartz. Phys. Rev. 121, 1324-1335 (1961). 


\section{Supplementary Files}

This is a list of supplementary files associated with this preprint. Click to download.

- PasslerHyperbolicShearPolaritonsinLowSymmetryCrystalsSupplementaryInformation.docx 